medir la glucemia y el perfil lipídico. Además, se recabaron los datos demográficos, se midió la circunferencia en la cintura y la presión arterial y se aplicó el cuestionario específico para medir la calidad de vida durante la menopausia denominado MENQOL.

Los síntomas encontrados con mayor frecuencia fueron las oleadas de calor $(53,3 \%)$, la sudoración $(49,2 \%)$, la pérdida de memoria $(80,6 \%)$, la depresión $(67,4 \%)$, lo dolores musculares y articulares $(84,0 \%)$, la resequedad de la piel $(85,5 \%)$, la evasión a la intimidad $(76,2 \%)$ y los cambios en el deseo sexual $(76,5 \%)$. La población estudiada presentó algunas similitudes y diferencias, en comparación con las mujeres menopáusicas de otros grupos étnicos. Esas diferencias deben estudiarse con mayor profundidad, aunque las características socioeconómicas y demográficas pueden explicar algunas de ellas. Por ejemplo, la paridad, los síntomas vasomotores y los trastornos sexuales no estuvieron relacionados con una mayor tasa de síntomas menopáusicos en este estudio, como se ha observado en otros estudios realizados en mujeres ecuatorianas de bajos ingresos.

Durante la transición menopáusica aparecen algunas manifestaciones relacionadas con el síndrome metabólico — como la hipertensión, la hiperinsulinemia, el perfil lipídico aterogénico y la diabetes-, que a su vez aumentan el riesgo cardiovascular. En la población estudiada se encontró una mayor prevalencia de síndrome metabólico $(41,5 \%)$, hipertensión arterial $(38,8 \%)$, hiperglucemia $(16,6 \%)$, hipertrigliceremia $(56,9 \%)$ y obesidad abdominal $(54,2 \%)$, en comparación con otras poblaciones. La característica diagnóstica más importante encontrada en las mujeres con síndrome metabólico, según el criterio adoptado por el Tercer Panel de Tratamiento de Adultos (ATP III), fue la obesidad abdominal $(83,7 \%)$. Los niveles elevados de triglicéridos y la obesidad abdominal elevaron el riesgo de sufrir oleadas de calor, mientras que la mayor edad y el tener 5 años o más de menopausia lo redujeron. La hipertrigliceremia también incrementó el riesgo de sufrir sudoraciones.

Estos resultados confirmaron que la tasa de depresión en las mujeres postmenopáusicas que presentaban obesidad abdominal es mayor que en las no obesas, además de presentar dolores musculares $\mathrm{y}$ articulares en mayor grado. Las mujeres con niveles elevado de glucemia en ayunas ( $>110 \mathrm{mg} / \mathrm{dL}$ ) presentaron un mayor riesgo de resequedad de la piel y de cambios en su deseo sexual. La mayor edad también se asoció significativamente con una mayor frecuencia de resequedad de la piel.

En conclusión, la frecuencia de síntomas menopáusicos en esta población de mujeres ecuatorianas fue similar a la encontrada en otras poblaciones de mujeres latinoamericanas y de otras regiones y estuvo asociada con la edad y el estado hormonal y metabólico. Se observó una asociación significativa entre la obesidad abdominal y la presencia de síntomas menopáusicos. Se deben recomendar cambios en el estilo de vida y en los hábitos alimentarios, ya que constituyen medidas eficaces y sencillas para mejorar la calidad de vida y reducir el riesgo cardiovascular en las mujeres postmenopáusicas. (Chedraui P, Hidalgo L, Chávez D, Morocho N, Alvarado $\mathrm{M}$, Huc A. Menopausal symptoms and associated risk factors among postmenopausal women screened for the metabolic syndrome. Arch Gynecol Obstet. 2007;275:161-8.)

\section{Adaptación al español de una escala de evaluación del miedo a la muerte}

Los cuestionarios para medir la ansiedad ante la muerte deben medir las diversas dimensiones de la actitud ante la muerte. La ansiedad es un concepto multidimensional que puede tener diversas causas, lo que lleva a las personas a reaccionar de forma diferente ante la idea de la muerte como un estado y de la muerte como proceso. De igual manera, la actitud y las reacciones emocionales pueden diferir cuando se trata de la muerte propia o de la ajena. La escala de miedo a la muerte de ColletLester (EMMCL) es uno de los instrumentos multidimensionales clásicos empleados para evaluar la actitud ante la muerte y es el único que permite distinguir entre la muerte y el proceso de morir, tanto con respecto a la muerte propia como a la ajena. Para ello, esta escala está estructurada en cuatro subescalas específicas independientes.

En este trabajo se expone el proceso de adaptación de la EMMCL al español, se determinan sus propiedades psicométricas (consistencia interna, confiabilidad y estructura factorial) y se establece la correlación de esa escala con otras que miden la ansiedad ante la muerte y la ansiedad en general.

Inicialmente, dos expertos bilingües tradujeron por separado las 28 preguntas de la EMMCL del inglés al español. Después, dos sicólogos revisaron la traducción y se sucedieron ciclos de correcciones y revisiones hasta lograr una versión definitiva de consenso. Luego se tradujo esa versión definitiva del español al inglés y se comparó con la versión original en inglés. Ambas versiones mostraron una gran similitud.

Para su validación, la versión final en español se aplicó a 109 enfermeras graduadas (tasa de respuesta: 76,43\%) y 172 estudiantes de enfermería (tasa de respuesta: 96,22\%), todos españoles; de ellos 64 hombres y 217 mujeres. La edad media de las enfermeras fue de 36,4 años (desviación están- 
dar: 8,3 años) y la de los estudiantes, de 23,1 años (desviación estándar: 5,0 años).

De acuerdo con los resultados obtenidos, se puede concluir que la versión española de la EMMCL presentó buenas propiedades psicométricas, es decir, tuvo una buena consistencia interna y una aceptable estabilidad temporal. La correlación de las cuatro subescalas de la EMMCL con las escalas de ansiedad ante la muerte y de ansiedad general fue similar a la obtenida en otros estudios, lo que demuestra la validez convergente de esta escala. Se debe resaltar especialmente que las correlaciones de las cuatro subescalas de la EMMCL con la ansiedad ante la muerte fueron mayores que las encontradas con las escalas de ansiedad general, lo que denota la validez discriminante de la escala española.

Siguiendo un patrón similar, los componentes extraídos ofrecieron un respaldo considerable a la validez factorial de la EMMCL. En este estudio, la carga en los cuatro componentes fue congruente con el factor hipotético de la construcción original de la escala en $93 \%$ de los casos, en particular, de los componentes de miedo a la muerte propia y de miedo a morir uno mismo. Estos resultados son es- pecialmente importantes debido al hecho de que en estudios anteriores que utilizaron las versiones original y árabe, la carga de los factores no se correspondió con los cuatro factores teóricos. Sin embargo, en la mayoría de los estudios anteriores, las muestras empleadas estuvieron conformadas por estudiantes de ciencias sociales o por población general.

Los resultados de la validación demuestran que la versión española de la EMMCL puede servir para identificar los miedos más frecuentes y evaluar los cambios subsecuentes. El hecho de que esta escala permita distinguir entre el miedo a la muerte (como estado) y el miedo a morir (como proceso), la hace apropiada para aplicarse en el campo de la salud, donde el contacto profesional con la muerte y lo relacionado con ella forma parte de la actividad cotidiana. Queda por comprobar su posible aplicación en personas hispanoparlantes fuera de España. La versión final de la EMMCL en español se presenta como apéndice en este artículo. (TomásSábado J, Limonero JT, Abdel-Khalek AM. Spanish adaptation of the Collett-Lester fear of death scale. Death Stud. 2007;31:249-60.) 